\title{
Mitochondrial Lon protease-unique structure and essential function in mammalian cells
}

\author{
Eva Kutejová* \\ Department of Biochemistry and Protein Structure, Institute of Molecular Biology, Slovak Academy of Sciences, Bratislava, Slovakia
}

\section{Editorial}

Lon protease belongs to the family of ATP dependent proteases. The Lon protease was first characterized by Prof. Alfred Goldberg in E. coli in 1981 [1]. Since then, it has been found in almost all living cells except some parasites like Trypanosoma brucei. In eukaryotes Lon is localized mainly in mitochondria, however peroxisomes as well as chloroplasts have their own Lon proteases. Although several eukaryotes can exist without Lon protease, for mammals it is an essential enzyme whose dysfunction has been identified in a number of diseases. Downregulation of this key mitochondrial component was observed in association with aging. Lon protease plays an important role also in malignant transformation that requires the adaptation to new sources of energy and is connected to hypoxia. This is the reason why Lon is highly expressed in aggressive tumors [2].

How does this remarkable protease function? It uses ATP to promote change to its structure, substrate unfolding and substrate translocation to its specific proteolytic cavity for degradation. Substrate recognition and degradation by Lon substantially differs from other proteolytic enzymes. It does not just recognize a specific cleavage site, but the first cleavage properly folded, functionally competent mitochondrial proteins occurs preferentially between hydrophobic amino acids located within highly charged regions [3]. Lon protease also possesses a chaperone activity, although not like other ATPdependent proteases. It cannot unfold already aggregated proteins but can participate in assembling of some OXPHOS complexes like $\mathrm{F}_{1} \mathrm{~F}_{0}$ ATPase and cytochrome $c$ oxidase [4]. Lon cooperates with other mitochondrial chaperones and protects mitochondria against oxidative stress. In mitochondria it is localized in mitochondrial matrix and within mitochondrial nucleoid which reflects its protease activity. In matrix, it is responsible for degrading oxidatively damaged proteins [5] and also regulates the level of several proteins or protein subunits, thus maintaining the equilibrium between imported and mitochondrially synthesized proteins and protein components. In mitochondrial nucleoid it guarantees the stability of mitochondrial DNA and at the same time facilitates the dynamics of this large protein - DNA complex to allow replication and transcription of mitochondrial DNA. Lon also directly binds mitochondrial DNA [6] specifically to G-rich regions that can form G-quadruplex secondary structures. The precise role of this characteristic is not known yet.

The function and level of Lon protease is in vivo tightly regulated in several different ways and in mammals appears to be organ specific. This crucial protease is regulated on the level of expression, posttranslational modification and interaction with specific partners like chaperones. Moreover, it significantly depends upon conditions in which the organism is located.

Although Lon protease might be considered the simplest ATPdependent protease, consisting of only identical subunits, six in humans and seven in yeast, this is not the case. Cryo-electron microscopy studies suggest that Lon has a flexible structure which undergoes rearrangement when either ATP or ADP is bound. Recently the 3D structure of human Lon has shown that both protease and ATPase domains form a barrellike "head" with pseudo six-fold symmetry. The "head" narrows into a tight trimeric neck and continues as six Lon monomers forming three pairs of "legs", belonging to the N-terminal domain, emerging from the neck as a trimer of dimers. The manifold arrangements of the existing structures of Lon's AP-domain hexamer observed in the presence of an ATP analogue and ADP reflect its flexibility, which is necessary for its allosteric operations [7].

Daniela A. Bota and Kelvin J. A. Davies [5] called Lon protease a Janus-like protein. This association perfectly fits its function. Lon belongs to the guardians of the healthy and vital cells. Any dysregulation of such key enzyme has severe consequences for the homeostasis of cells and leads either to pathological conditions such as cancer or aging and cell death.

\section{Acknowledgment}

The author has received support from the Slovak Grant Agency (VEGA 2/0075/18) and from the Slovak Research and Development Agency (APVV-15-0375). Many thanks also to Nina Kunová and Jacob Bauer for helpful comments.

\section{References}

1. Chung CH, Goldberg AL (1981) The product of the lon (capR) gene in Escherichia coli is the ATP-dependent protease, protease La. Proc Natl Acad Sci U S A 78: 4931-4935. [Crossref]

2. Gibellini L, Losi L, De Biasi S, Nasi M, Lo Tartaro D, et al. (2018) LonP1 Differently Modulates Mitochondrial Function and Bioenergetics of Primary Versus Metastatic Colon Cancer Cells. Front Oncol 8: 254. [Crossref]

3. Ondrovicová G, Liu T, Singh K, Tian B, Li H, et al. (2005) Cleavage site selection within a folded substrate by the ATP-dependent lon protease. J Biol Chem 280: 2510325110. [Crossref]

*Correspondence to: Eva Kutejová, Department of Biochemistry and Protein Structure, Institute of Molecular Biology, Slovak Academy of Sciences, Bratislava, Slovakia, Tel: 00421259307444, E-mail: Eva.Kutejova@savba.sk

Received: December 08, 2018; Accepted: December 18, 2018; Published: December 22, 2018 
4. Rep M, van Dijl JM, Suda K, Schatz G, Grivell LA, et al. (1996) Promotion of mitochondrial membrane complex assembly by a proteolytically inactive yeast Lon. Science 274: 103-106. [Crossref]

5. Bota DA, Davies KJ (2016) Mitochondrial Lon protease in human disease and aging: Including an etiologic classification of Lon-related diseases and disorders. Free Radic Biol Med 100: 188-198. [Crossref]
6. Ambro L, Pevala V, Bauer J, Kutejová E (2012) The influence of ATP-dependent proteases on a variety of nucleoid-associated processes. J Struct Biol 287: 2245022462. [Crossref]

7. Kereïche S, Kovácik L, Bednár J, Pevala V, Kunová N, et al. (2016) The N-terminal domain plays a crucial role in the structure of a full-length human mitochondrial Lon protease. Sci Rep 6: 33631.

Copyright: (C2018 Kutejová E. This is an open-access article distributed under the terms of the Creative Commons Attribution License, which permits unrestricted use, distribution, and reproduction in any medium, provided the original author and source are credited. 